The following resources related to this article are available online at www.sciencemag.org (this information is current as of December 7, 2007 ):

Updated information and services, including high-resolution figures, can be found in the online version of this article at:

http://www.sciencemag.org/cgi/content/full/309/5731/56c

A list of selected additional articles on the Science Web sites related to this article can be found at:

http://www.sciencemag.org/cgi/content/full/309/5731/56c\#related-content

This article cites 2 articles, 1 of which can be accessed for free:

http://www.sciencemag.org/cgi/content/full/309/5731/56c\#otherarticles

This article appears in the following subject collections:

Psychology

http://www.sciencemag.org/cgi/collection/psychology

Technical Comments

http://www.sciencemag.org/cgi/collection/tech_comment

Information about obtaining reprints of this article or about obtaining permission to reproduce this article in whole or in part can be found at:

http://www.sciencemag.org/about/permissions.dtl 


\section{Response to Comment on "Children Creating Core Properties of Language: Evidence from an Emerging Sign Language in Nicaragua"}

In our recent study (1), we argued that the analytical approach that children apply to language learning can generate fundamental design features of language. We observed combinatorial linear structure within motion event expressions, particularly in those who learned Nicaraguan Sign Language (NSL) after 1984, when it was passed down to a new generation of children. We concluded that intrinsic properties of young children's learning abilities have contributed discreteness and combinatorial organization to NSL over the past 25 years (2).

In their comment, Russo and Volterra (3) raise three methodological concerns that imply that our analyses overlooked certain features of the learning environment. Candidate environmental sources of the observed structure must satisfy two conditions: They must present an accessible model of the structure, and they must have become available to learners only after 1984. As we argue below, the alternative sources that Russo and Volterra propose satisfy neither condition.

Russo and Volterra's first question concerns participants' exposure to Spanish in vocal or written form before coming into contact with other deaf children. The vocal and written Spanish exposure afforded to young deaf children in Nicaragua appears not to have changed over the past 25 years. Both are minimally available. Few of our participants received hearing aids as children, and none received audiological training or speech therapy. Children arrive at school at age 4 or 5 speaking a few isolated Spanish words and apparently have never "read" through even a picture book (4). Spanish is taught in school, but by graduation at the completion of sixth grade, none of the students have an easy command of written or spoken Spanish.

Furthermore, if Spanish were the source of the structures we observed in NSL, the two languages should show similarities in their linear orders. We do not observe such similarities. For example, in NSL, manner typically precedes path (roll descend), whereas in Spanish, path precedes manner (descend rolling). The A-B-A construction frequently observed in NSL (roll descend roll) is quite unlike any Spanish construction. It thus appears unlikely that the structures we documented derive from exposure to Spanish in any form.
The second proposed source of structure is gesture produced in the absence of speech. Two studies have examined the structure of motion events in gestures without speech: GoldinMeadow, McNeill, and Singleton (5) with English speakers, and Özyürek and GoldinMeadow (6) with Spanish speakers. Both studies found that aspects of motion events (such as figure, manner, and path) are included in a single gesture rather than segmented into separate gestures $(7,8)$. Thus, even if Nicaraguan parents did communicate with their deaf children using gesture without speech, such gestures are unlikely to be a source of the segmented and sequenced signing that has emerged in NSL.

The final source of structure proposed by Russo and Volterra is mouth movement. In a follow-up analysis, we first examined whether signers brought Spanish segmentation into their signing via mouthed Spanish. Two independent coders recoded one-third of the deaf participants' motion event expressions with respect to whether a Spanish word was mouthed (9). The only Spanish mouthing observed were two instances of the Spanish noun for cat (gato) mouthed simultaneously with the production of the manual sign for cat. No mouthing of Spanish words for manner or path accompanied the NSL signs for motion. Thus, it seems unlikely that the segmentation of manner and path derives from structure imposed by mouthing Spanish.

Additionally, although participants frequently produced mouth movements that indicated an aspect of the manner of movement (e.g., repeated, effortful), these always accompanied a manual movement. Precisely because they are always produced simultaneously with a manual sign, mouth movements never exhibit the linear sequencing being documented in this study.

The emergence of NSL provides a rare opportunity to study the origins of linguistic structure in vivo. This new language did not come from "nowhere"; it arose in a womb of rich human interaction. We agree with Russo and Volterra that emergent languages should be closely evaluated, case by case. In this case, we selected a definitive component of language and documented its growth by comparing its use by older and younger signers. The design is simple, but the change is not. The segmentation of manner and path poses a stringent test of segmentation. The data show that this discrete structure, this hallmark of language, emerged in Nicaraguan signing as it was passed down for the first time to a new generation of learners. This is the way a new language is born.

Ann Senghas
Department of Psychology
Barnard College of Columbia University
3009 Broadway
New York, NY 10027, USA
Asli Özyürek
F. C. Donders Center for
Cognitive Neuroimaging
Nijmegen University
Adelbertusplein 1

6525 EK Nijmegen, The Netherlands and Max Planck Institute for Psycholinguistics Wundtlaan 1 6525 XD Nijmegen, The Netherlands and Koç University Department of Psychology Rumeli Feneri Yolu 34450, Sariyer, Istanbul, Turkey

Sotaro Kita

Department of Experimental Psychology University of Bristol 8 Woodland Road Bristol BS8 1TN, UK

References and Notes

1. A. Senghas, S. Kita, A. Özyürek, Science 305, 1779 (2004).

2. We argue that these changes are consequences of children's learning processes. We do not take a position as to whether the processes involved in these changes are language specific or are also applied generally.

3. T. Russo, V. Volterra, Science 309, 56 (2005); www.sciencemag.org/cgi/content/full/309/5731/56b.

4. This minimal exposure to text is not limited to deaf children; it is characteristic of the larger Nicaraguan culture.

5. S. Goldin-Meadow, D. McNeill, J. Singleton, Psychol. Rev. 103, 34 (1996)

6. A. Özyürek, S. Goldin-Meadow, in preparation.

7. NSL is not the only case in which unsegmented input was segmented by child learners. Segmentation and sequencing of motion events have been observed in home signers exposed only to gesture that accompanies speech. For example, Goldin-Meadow reports that the home signer she calls David produced the sequence flutter descend to describe snowing [figure 3 in (8)]. David's mother never produced sequenced combinations of this or any other type.

8. S. Goldin-Meadow, The Resilience of Language: What Gesture Creation in Deaf Children Can Tell Us About How All Children Learn Language (Psychology Press, New York, 2004).

9. Intercoder reliability yielded agreement of 1.0.

10. We thank A. Engelman and M. Flaherty for assistance with data analysis. Supported by the Max Planck Institute for Psycholinguistics, Netherlands Organization for Scientific Research (NWO) project 051.02.040 (A.Ö.), National Institute on Deafness and Other Communication Disorders (NIH/NIDCD) grant R01 DC00491 (S. Goldin-Meadow and A.Ö.), Turkish Academy of Sciences grant HAO/TUBAGEBIP/2001-2-16 (A.O.), and NIH/NIDCD grant R01 DC05407 (A.S.).

10 February 2005; accepted 27 May 2005 10.1126/science. 1110901 